\title{
First-line chemotherapy with S-1 alone or S-1 plus cisplatin for elderly patients with advanced gastric cancer: a multicenter propensity score matched study
}

\author{
Akitaka Makiyama $^{1} \cdot$ Kenji Kunieda $^{2} \cdot$ Masaaki Noguchi $^{3} \cdot$ Takeshi Kajiwara $^{4} \cdot$ Takao Tamura $^{5} \cdot$ Koji Takeda $^{6}$. \\ Junko Sugiyama $^{7} \cdot$ Keiko Minashi $^{8} \cdot$ Toshikazu Moriwaki $^{9} \cdot$ Naotoshi Sugimoto $^{10} \cdot$ Michitaka Nagase $^{11}$. \\ Yuji Negoro ${ }^{12}$. Takashi Tsuda ${ }^{13}$. Hideki Shimodaira ${ }^{14} \cdot$ Naohiro Okano $^{15} \cdot$ Akihito Tsuji $^{16}$. Daisuke Sakai ${ }^{17}$. \\ Kazuhiro Yanagihara ${ }^{18}$. Shinya Ueda ${ }^{19}$. Shingo Tamura ${ }^{20}$. Satoshi Otsu ${ }^{21} \cdot$ Takuya Honda $^{22}$. Yuzo Matsushita ${ }^{23}$. \\ Tatsuya Okuno ${ }^{24} \cdot$ Tomomi Kashiwada $^{25} \cdot$ Akira Nozaki $^{26} \cdot$ Masahide Ebi $^{27} \cdot$ Hiroyuki Okuda $^{28}$. \\ Mototsugu Shimokawa ${ }^{29}$. Shuichi Hironaka ${ }^{8}$. Ichinosuke Hyodo ${ }^{9} \cdot$ Eishi Baba $^{30} \cdot$ Narikazu Boku $^{31} \cdot$ Kei Muro $^{32}$. \\ Taito Esaki ${ }^{33}$
}

Received: 3 October 2017 / Accepted: 14 January 2018 / Published online: 20 January 2018

(c) The International Gastric Cancer Association and The Japanese Gastric Cancer Association 2018

\begin{abstract}
Background Fluoropyrimidine and platinum combination is the standard treatment for advanced or recurrent gastric cancer (AGC). However, fluoropyrimidine monotherapy is commonly used for elderly patients with AGC because of its good tolerability.

Methods In this multicenter retrospective study, we collected clinical data of AGC patients aged 70 years or older, treated with S-1 alone or S-1 plus cisplatin (SP) as the first-line treatment between January 2009 and December 2011. Propensity score matched cohorts (PSMC) were used for reducing the confounding effects to compare efficacy and safety between the two treatment groups. Cox regression analysis was performed to clarify the prognostic factors.

Results PSMC ( $n=109$ in each group) were selected from among 444 eligible patients (S-1 group, 210; SP group, 234); the S-1 group included more patients deemed unfit for intensive chemotherapy than the SP group (e.g., higher age, poorer PS, poor renal function). In the PSMC, patients' characteristics were comparable between groups, except the male ratio (S-1 group, 64.2\%; SP group, 77.1\%; $\mathrm{p}=0.04$ ). No significant differences were observed in either overall survival [hazard ratio (HR) $0.93, p=0.63$ ] or progression-free survival (HR 1.09, $p=0.61$ ). Severe adverse events (AEs) and hospitalization due to AEs were more frequent in the SP group than in the S-1 group $(p<0.001$ each).

Conclusion Our findings do not support the survival benefit of SP over S-1 in elderly patients with AGC. We are now conducting a prospective comparative study to optimize treatment strategy and explore applicability of the geriatric assessment for these patients.
\end{abstract}

Keywords Elderly patients $\cdot$ Gastric cancer $\cdot$ S- 1 - Cisplatin $\cdot$ Propensity score matching method (3-5 words)

Electronic supplementary material The online version of this article (https://doi.org/10.1007/s10120-018-0797-y) contains supplementary material, which is available to authorized users.

Akitaka Makiyama

makiyama20@hotmail.com

Extended author information available on the last page of the article

\section{Introduction}

Gastric cancer is the fourth most common cancer and the second leading cause of cancer deaths worldwide [1]. Recently, the number of elderly patients with gastric cancer has increased. In 2010, the incidence of gastric cancer in patients aged 70 years or older was estimated to be 75,000 per year, which accounted for $60 \%$ of the total number of patients with this cancer in Japan [2]. The mortality rate in elderly Japanese patients in 2013 was estimated to be 35,000 per year, which accounted for approximately $70 \%$ of the total 
deaths among patients with gastric cancer [2]. NCCN guideline for older adult oncology describes approach to decision making in cancer treatment for elderly patients [3]. In this approach, assessment of capability to make decision and patient's treatment goal and value are most important. On the other hand, the standard chemotherapy for young patients might be very toxic for the elderly patients because of organ dysfunction or comorbidity even if they are regarded as tolerable for chemotherapy.

Doublet chemotherapy using fluoropyrimidine and platinum have been worldwide used as first-line regimens for patients with advanced gastric cancer (AGC) [4]. In Japan, doublet chemotherapy with S-1 plus cisplatin (SP) has been recognized as the standard first-line chemotherapy regimen for advanced gastric cancer based on the results of phase III trials [5, 6]. Patients over 75 years of age were excluded from these trials. Although a subgroup analysis of patients aged under 60 years in the SPIRITS trial that compared S-1 with SP showed better overall survival (OS) in the SP group [hazard ratio (HR) $0.75,95 \%$ confidence interval (CI) 0.61-0.92], the other subgroups of patients aged 60-69 years (HR 0.98, 95\% CI 0.82-1.17) and 70-74 years (HR 0.95, 95\% CI 0.71-1.27) demonstrated similar survival between the S-1 and SP groups [6]. Two small retrospective studies comparing S-1 with SP for the patients with AGC older than 70 years have also been reported without adjusting the effects of confounding factors [7, 8]. While one study showed better efficacy of SP, the other did not, although both studies reported that SP showed more severe toxicities than $\mathrm{S}-1$. These findings suggest that the addition of cisplatin to S-1 may not be beneficial compared with S-1 alone in elderly patients with AGC, especially those aged 70 years or older. To date, large-scale randomized study for elderly patients with AGC has not been conducted, and standard therapy has yet to be established.

In this multicenter retrospective study, to explore the feasible treatment for elderly patients with AGC, we compared efficacy and safety between S- 1 and SP regimens using propensity score matched analysis that can reduce the confounding effects of patients' background factors.

\section{Patients and methods}

\section{Patients}

The subjects of this study were registered and retrospectively reviewed from patients with AGC who received the first-line chemotherapy in the clinical practice setting between January 2009 and December 2011 at the 29 medical centers participating in the West Japan Oncology Group (WJOG). The selection criteria were: (1) aged 70 years or older; (2) histologically proven metastatic or recurrent gastric adenocarcinoma; (3) measurable or assessable lesion according to the Response Evaluation Criteria in Solid Tumor (RECIST) version 1.1 [9]; (4) Performance Status (PS) score of $0-2$; (5) treatment with S-1 monotherapy or SP; and (6) no prior chemotherapy, except adjuvant chemotherapy with S- 1 alone completed more than 24 weeks prior to the confirmation of relapse. Patients who received trastuzumab, and those with active multiple primary cancers, brain metastasis, and massive pleural effusion or ascites, requiring intensive treatment, were excluded from the study. The present study was approved by the institutional review board in each institution.

\section{Treatment}

All patients received systemic chemotherapies with SP or S-1 as the first-line treatment. Patients in the S-1 group received S-1 (40-60 mg, twice daily, depending on the body surface area of the patient) administered orally on days 1-28, repeated every 6 weeks. Patients in the SP group received the same dose of S-1 administered orally on days 1-21, and $60 \mathrm{mg} / \mathrm{m}^{2}$ cisplatin on day 8 , repeated every 5 weeks. Dose reductions, treatment delays, and modification of treatment schedule (i.e., S-1 administered orally from day 1-14 every 3 weeks) were performed by physician's decision considering adverse events (AEs). The treatments were continued until disease progression, unacceptable toxicity, or patient refusal.

\section{Assessments}

Collected data regarding patients' characteristics before the initiation of chemotherapy included age, sex, PS, laboratory tests, diseases status (recurrent or unresectable), prior gastrectomy, metastatic sites, and histological type. For the assessment of the vulnerability of the elderly patients, comorbidities and any relevant medications were surveyed. The administration doses, schedules of S-1 and SP treatments, treatment efficacy, and AEs were reviewed. The tumor response was assessed using computed tomography, gastrointestinal endoscopy, and/or magnetic resonance imaging. Response was evaluated according to the RECIST ver. 1.1 by the physician of each institution. The worst grade for each $\mathrm{AE}$ during the entire treatment period was evaluated according to the Common Terminology Criteria of Adverse Events ver. 4.0. The reasons for the termination of each drug and the subsequent therapies were also reviewed.

\section{Statistics}

Patients' characteristics were summarized using descriptive statistics or contingency tables. A Chi square test was used to compare the variables between the two groups. 
A propensity score method was applied to reduce selection bias for comparisons between the treatment arms, and logistic regression model was used to calculate propensity scores for the patients. Propensity score matching was performed in the ratio 1:1 using a caliper width of 0.1. OS, progression-free survival (PFS), and time to treatment failure (TTF) curves were estimated by the Kaplan-Meier method, and their 95\% CIs were estimated using the Brookmeyer and Crowley method. The prognostic factors for OS were assessed using the Cox proportional hazards models with backward elimination method. OS was defined as the period from the date of chemotherapy initiation to the date of death from any cause. PFS was defined as the period from the date of chemotherapy initiation to the date of tumor progression or death from any cause. TTF was defined as the period from the initiation of the therapy to the last day of administration of S-1. $P$ values correspond to two-sided tests, and statistical significance was considered as $<0.05$. Factors included in multivariate analysis for adjusting patient background, OS, and PFS are listed in Appendix Table 1. Statistical analyses were performed using SAS 9.4 software (SAS Institute, Cary, NC, USA) and IBM SPSS Statistics for Windows, version 23 (IBM Corp., Armonk, N.Y., USA).

\section{Results}

\section{Patients' characteristics of the original cohort and propensity score matched cohort (PSMC)}

A total of 464 patients with AGC were initially registered; among whom 20 were excluded for the following reasons: treatment outside of the pre-specified period $(n=10)$, ineligible treatment history $(n=3)$, unknown histology $(n=2)$, unknown PS $(n=1)$, double cancer $(n=1)$, duplicate registration $(n=1)$, less than 70 years of age $(n=1)$, and lost to follow-up $(n=1)$. Finally, 444 patients were included in the analysis (Fig. 1).

S-1 or SP was administered to $210(47.3 \%)$ and 234 $(52.7 \%)$ patients, respectively (Table 1$)$. The median age of S-1 group and SP group were 77 and 73 years, respectively. The number of patients aged 70-74 years was $56(26.7 \%)$ in the S-1 group, and $163(69.7 \%)$ in the SP group $(p<0.001)$. The S-1 group included more patients with unfavorable PS $(p<0.001)$, comorbidities $(p<0.001)$, medications for comorbidities $(p<0.05)$, low renal function $(p<0.01)$, and prior gastrectomy $(p<0.01)$ than the SP group. Additionally, peritoneal metastasis was more frequently observed in the SP group than in the $S-1$ group $(p<0.01)$. Patients in the $\mathrm{S}-1$ group did not receive cisplatin for the following reasons: older age $(54.3 \%)$, low creatinine clearance (11.4\%), poor PS (7.6\%), comorbidities (7.1\%), and other reasons
Fig. 1 Patient flow diagram. $S P$ S-1 plus cisplatin, $P S$ Eastern Cooperative Oncology Group performance status

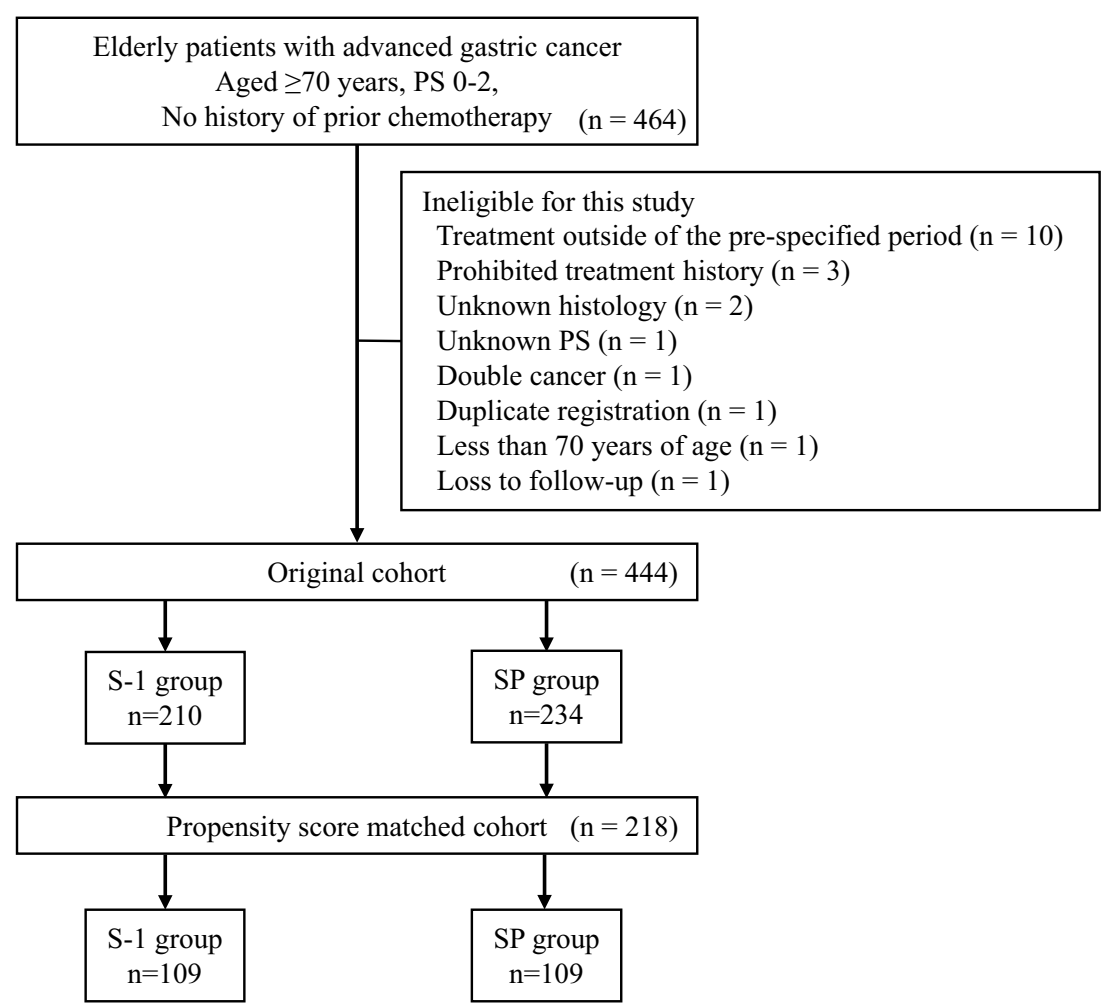


Table 1 Patients' characteristics of the original cohort and propensity score matched cohort

\begin{tabular}{|c|c|c|c|c|c|c|c|c|c|c|}
\hline \multirow[t]{4}{*}{ Characteristics } & \multicolumn{5}{|c|}{ Original cohort } & \multicolumn{5}{|c|}{ Propensity score matched cohort } \\
\hline & \multicolumn{2}{|l|}{ S-1 } & \multicolumn{3}{|l|}{ SP } & \multicolumn{2}{|l|}{ S-1 } & \multicolumn{3}{|l|}{ SP } \\
\hline & \multicolumn{2}{|l|}{$(n=210)$} & \multicolumn{3}{|l|}{$(n=234)$} & \multicolumn{2}{|l|}{$(n=109)$} & \multicolumn{3}{|l|}{$(n=109)$} \\
\hline & $n$ & $(\%)$ & $n$ & $(\%)$ & $p$ value & $n$ & $(\%)$ & $n$ & $(\%)$ & $p$ value \\
\hline \multicolumn{11}{|l|}{ Age (years) } \\
\hline Median (range) & 77 & $(70-86)$ & 73 & $(70-86)$ & & 76 & $(70-86)$ & 75 & $(70-84)$ & \\
\hline$<75$ & 56 & $(26.7)$ & 163 & $(69.7)$ & & 48 & $(44.0)$ & 48 & $(44.0)$ & \\
\hline$\geq 75$ & 154 & $(73.3)$ & 71 & $(30.3)$ & $<0.001$ & 61 & $(56.0)$ & 61 & $(56.0)$ & 1.00 \\
\hline \multicolumn{11}{|l|}{ Sex } \\
\hline Male & 142 & $(67.6)$ & 174 & $(74.4)$ & & 70 & $(64.2)$ & 84 & $(77.1)$ & \\
\hline Female & 68 & $(32.4)$ & 60 & $(25.6)$ & 0.12 & 39 & $(35.8)$ & 25 & $(22.9)$ & 0.04 \\
\hline \multicolumn{11}{|l|}{ Performance status } \\
\hline 0 & 56 & $(26.7)$ & 114 & $(48.7)$ & & 42 & $(38.5)$ & 43 & $(39.4)$ & \\
\hline$\geq 1$ & 154 & $(73.3)$ & 120 & $(51.3)$ & $<0.001$ & 67 & $(61.5)$ & 66 & $(60.6)$ & 0.89 \\
\hline \multicolumn{11}{|l|}{ Body mass index } \\
\hline Median & 20.8 & & 20.9 & & & 20.4 & & 21.0 & & \\
\hline Range & $13.6-34.1$ & & $15.4-30.9$ & & & $14.1-31.2$ & & $15.4-27.4$ & & \\
\hline \multicolumn{11}{|c|}{ Creatinine clearance } \\
\hline$<50 \mathrm{ml} / \mathrm{min}$ & 56 & $(26.7)$ & 35 & $(15.0)$ & & 27 & $(24.8)$ & 27 & $(24.8)$ & \\
\hline$\geq 50 \mathrm{ml} / \mathrm{min}$ & 152 & $(72.4)$ & 199 & $(85.0)$ & & 82 & $(75.2)$ & 82 & $(75.2)$ & \\
\hline Unknown & 2 & $(1.0)$ & 0 & $(0.0)$ & $<0.01$ & 0 & $(0.0)$ & 0 & $(0.0)$ & 1.00 \\
\hline \multicolumn{11}{|c|}{ Number of comorbidity } \\
\hline Median (range) & $1(0-5)$ & & $1(0-5)$ & & & $1(0-5)$ & & $1(0-5)$ & & \\
\hline 0 & 50 & $(23.8)$ & 91 & $(38.9)$ & & 33 & $(30.3)$ & 32 & $(29.4)$ & \\
\hline 1 & 82 & $(39.0)$ & 69 & $(29.5)$ & & 40 & $(36.7)$ & 36 & $(33.0)$ & \\
\hline$\geq 2$ & 78 & $(37.1)$ & 74 & $(31.6)$ & $<0.01$ & 36 & $(33.0)$ & 41 & $(37.6)$ & 0.76 \\
\hline \multicolumn{11}{|l|}{ Comorbidity } \\
\hline Yes & 160 & $(76.2)$ & 143 & $(61.1)$ & & 76 & $(69.7)$ & 77 & $(70.6)$ & \\
\hline No & 50 & $(23.8)$ & 91 & $(38.9)$ & $<0.001$ & 33 & $(30.3)$ & 32 & $(29.4)$ & 0.88 \\
\hline \multicolumn{11}{|c|}{ Medication for comorbidity } \\
\hline Yes & 138 & $(65.7)$ & 127 & $(54.3)$ & & 67 & $(61.5)$ & 66 & $(60.6)$ & \\
\hline No & 72 & $(34.3)$ & 107 & $(45.7)$ & $<0.05$ & 42 & $(38.5)$ & 43 & (39.4) & 0.90 \\
\hline Histology & & & & & & & & & & \\
\hline Intestinal & 97 & $(46.2)$ & 105 & (44.9) & & 52 & $(47.7)$ & 48 & $(44.0)$ & \\
\hline Diffuse & 111 & $(52.9)$ & 126 & $(53.8)$ & & 57 & $(52.3)$ & 58 & $(53.2)$ & \\
\hline Others & 2 & $(1.0)$ & 3 & $(1.3)$ & 0.92 & 0 & $(0.0)$ & 3 & $(2.8)$ & 0.26 \\
\hline Prior gastrectomy & & & & & & & & & & \\
\hline Yes & 67 & $(31.9)$ & 49 & $(20.9)$ & & 35 & $(32.1)$ & 35 & $(32.1)$ & \\
\hline No & 143 & $(68.1)$ & 185 & $(79.1)$ & $<0.01$ & 74 & $(67.9)$ & 74 & $(67.9)$ & 1.00 \\
\hline Adjuvant chemoth & apy & & & & & & & & & \\
\hline Yes & 9 & $(4.3)$ & 12 & (5.1) & & 7 & (6.4) & 9 & (8.3) & \\
\hline No & 200 & $(95.2)$ & 222 & $(94.9)$ & & 101 & $(92.7)$ & 100 & $(91.7)$ & \\
\hline Unknown & 1 & $(0.5)$ & 0 & $(0.0)$ & 0.52 & 1 & $(0.9)$ & 0 & $(0.0)$ & 0.53 \\
\hline Number of metast & ic sites & & & & & & & & & \\
\hline Median (range) & $2(1-4)$ & & $2(1-4)$ & & & $2(1-4)$ & & $2(1-4)$ & & \\
\hline 1 & 92 & $(43.8)$ & 74 & $(31.6)$ & & 43 & $(39.4)$ & 40 & $(36.7)$ & \\
\hline 2 & 86 & $(41.0)$ & 113 & $(48.3)$ & & 50 & $(45.9)$ & 52 & $(47.7)$ & \\
\hline$\geq 3$ & 31 & (14.8) & 46 & (19.7) & & 15 & (13.8) & 16 & (14.7) & \\
\hline Unknown & 1 & $(0.5)$ & 1 & $(0.4)$ & 0.08 & 1 & $(0.9)$ & 1 & $(0.9)$ & 0.98 \\
\hline
\end{tabular}


Table 1 (continued)

\begin{tabular}{|c|c|c|c|c|c|c|c|c|c|c|}
\hline \multirow[t]{4}{*}{ Characteristics } & \multicolumn{5}{|c|}{ Original cohort } & \multicolumn{5}{|c|}{ Propensity score matched cohort } \\
\hline & \multicolumn{2}{|l|}{ S-1 } & \multicolumn{3}{|l|}{ SP } & \multicolumn{2}{|c|}{ S-1 } & \multicolumn{3}{|l|}{ SP } \\
\hline & \multicolumn{2}{|c|}{$(n=210)$} & \multicolumn{3}{|c|}{$(n=234)$} & \multicolumn{2}{|c|}{$(n=109)$} & \multicolumn{3}{|c|}{$(n=109)$} \\
\hline & $n$ & $(\%)$ & $n$ & $(\%)$ & $p$ value & $n$ & $(\%)$ & $n$ & $(\%)$ & $p$ value \\
\hline \multicolumn{11}{|l|}{ Target lesion } \\
\hline Yes & 159 & $(75.7)$ & 175 & $(74.8)$ & & 80 & $(68.4)$ & 87 & $(74.4)$ & \\
\hline No & 51 & $(24.3)$ & 59 & $(25.2)$ & 0.82 & 29 & $(24.8)$ & 22 & $(18.8)$ & 0.26 \\
\hline \multicolumn{11}{|c|}{ Site of metastasis } \\
\hline Liver & 86 & $(41.0)$ & 89 & $(38.0)$ & 0.53 & 45 & $(38.5)$ & 36 & $(30.8)$ & 0.21 \\
\hline Peritoneum & 82 & $(39.0)$ & 122 & $(52.1)$ & $<0.01$ & 49 & (41.9) & 49 & (41.9) & 1.00 \\
\hline Lung & 14 & $(6.7)$ & 15 & $(6.4)$ & 0.91 & 8 & $(6.8)$ & 8 & $(6.8)$ & 1.00 \\
\hline Lymph node & 155 & $(73.8)$ & 188 & $(80.3)$ & 0.10 & 75 & $(64.1)$ & 85 & (72.6) & 0.13 \\
\hline Bone & 6 & $(2.9)$ & 8 & $(3.4)$ & 0.74 & 3 & (2.6) & 3 & $(2.6)$ & 1.00 \\
\hline Others & 16 & (7.6) & 18 & (7.7) & 0.98 & 10 & (8.5) & 7 & (6.0) & 0.45 \\
\hline
\end{tabular}

SP S-1 + cisplatin

(14.8\%) (Appendix Table 2). Comorbidities were reported in 303 patients $(68.2 \%$ ) (Appendix Table 3). More patients in the SP group had hypertension $(p<0.01)$ than in the S-1 group. These data clearly show that there were substantial differences in the patients' backgrounds between the two groups. The median OS was 11.5 months in the S-1 group and 16.2 months in the SP group in the original entire cohort $(p=0.01)$.

Propensity scores were calculated using the five baseline variables; age ( 74 years or younger vs. 75 years or older), PS (0 vs. 1-2), comorbidities $( \pm)$, prior gastrectomy $( \pm)$, and peritoneal metastasis $( \pm)$, which differed significantly between the two groups. One hundred and nine patients in each group were matched by propensity score (Fig. 1). In the PSMC, there was no difference in patients' background between the two groups, apart from the sex ratio $(p=0.04)$ (Table 1).

\section{Treatments in the PSMC}

In the PSMC, the initial dose of S-1 was reduced in 47 patients $(43.1 \%)$ in the $\mathrm{S}-1$ group, whereas the initial dose of S-1 and cisplatin was reduced in 26 patients $(23.9 \%)$ and 30 patients $(27.5 \%)$ in the SP group, respectively (Appendix Table 4). The reasons for initial dose reduction of S-1 in the S-1 group were low creatinine clearance $(46.8 \%)$, older age $(34.0 \%)$, poor PS $(6.4 \%)$, comorbidities $(2.1 \%)$, and other reasons $(4.3 \%)$, whereas patients in the SP group required initial dose reduction for low creatinine clearance $(53.8 \%)$, older age $(19.2 \%)$, poor PS $(7.7 \%)$, and other reasons $(11.5 \%)$. Dose reduction of $\mathrm{S}-1$ was required in 26 patients $(23.9 \%)$ in the $\mathrm{S}-1$ group and 50 patients $(45.9 \%)$ in the SP group during the entire treatment period $(p<0.001)$, whereas 31 patients $(28.4 \%)$ in the SP group required dose reduction of cisplatin. Treatment was terminated in almost all patients $(95.9 \%)$.

The median TTF was 5.1 months in the S-1 group and 5.8 months in the SP group (HR 0.80, 95\% CI 0.60-1.06, $p=0.12$ ). The median time from the starting date of chemotherapy to the last dosage of cisplatin in the SP group was 3.7 months (95\% CI 2.5-4.8). The reasons for treatment discontinuation in the S-1 and SP groups were disease progression (70.6 and 73.4\%), AEs (11.9 and 11.0\%), patient refusal (4.6 and $1.8 \%$ ), worsening of comorbidity (0.9 and $0.9 \%)$, physician's decision (2.8 and 5.5\%), death during treatment $(0.9$ and $3.7 \%)$, and other reasons $(0.9$ and $2.8 \%)$, respectively (Appendix Table 5). Subsequent chemotherapies were administered to 46 patients of the S-1 group (42.2\%) and 72 patients of the SP group $(66.1 \%)(p<0.01)$ (Appendix Table 6).

\section{Efficacy in the PSMC}

The median follow-up time was 26.2 months $(95 \% \mathrm{CI}$ 25.5-27.0) in the S-1 group and 31.3 months (95\% CI 26.5-36.2) in the SP group. In the S-1 and SP groups, the overall response rates were 27.5 and $41.4 \%(p=0.06)$, and the disease control rates were 71.3 and $85.1 \%(p=0.03)$, respectively (Appendix Table 7). The median PFS was 6.3 months in the $\mathrm{S}-1$ group and 7.9 months in the SP group (HR 1.09, 95\% CI 0.79-1.49, $p=0.61$ ) (Fig. 2a). In the S-1 and SP groups, the PFS rate at 6 months was 53.1 and $59.7 \%$, and that at 12 months was 32.2 and $31.1 \%$, respectively. The median OS was 13.9 months in the $\mathrm{S}-1$ group and 17.1 months in the SP group (HR 0.93, 95\% CI 0.68-1.27, $p=0.63$ ) (Fig. 2b). Survival at 6 months was 82.5 and 
Fig. 2 Propensity score matched Kaplan-Meier survival analysis comparing the $\mathrm{S}-1$ group $(n=109)$ vs SP group $(n=109)$. a progression-free survival, b overall survival. SP, S-1 plus cisplatin
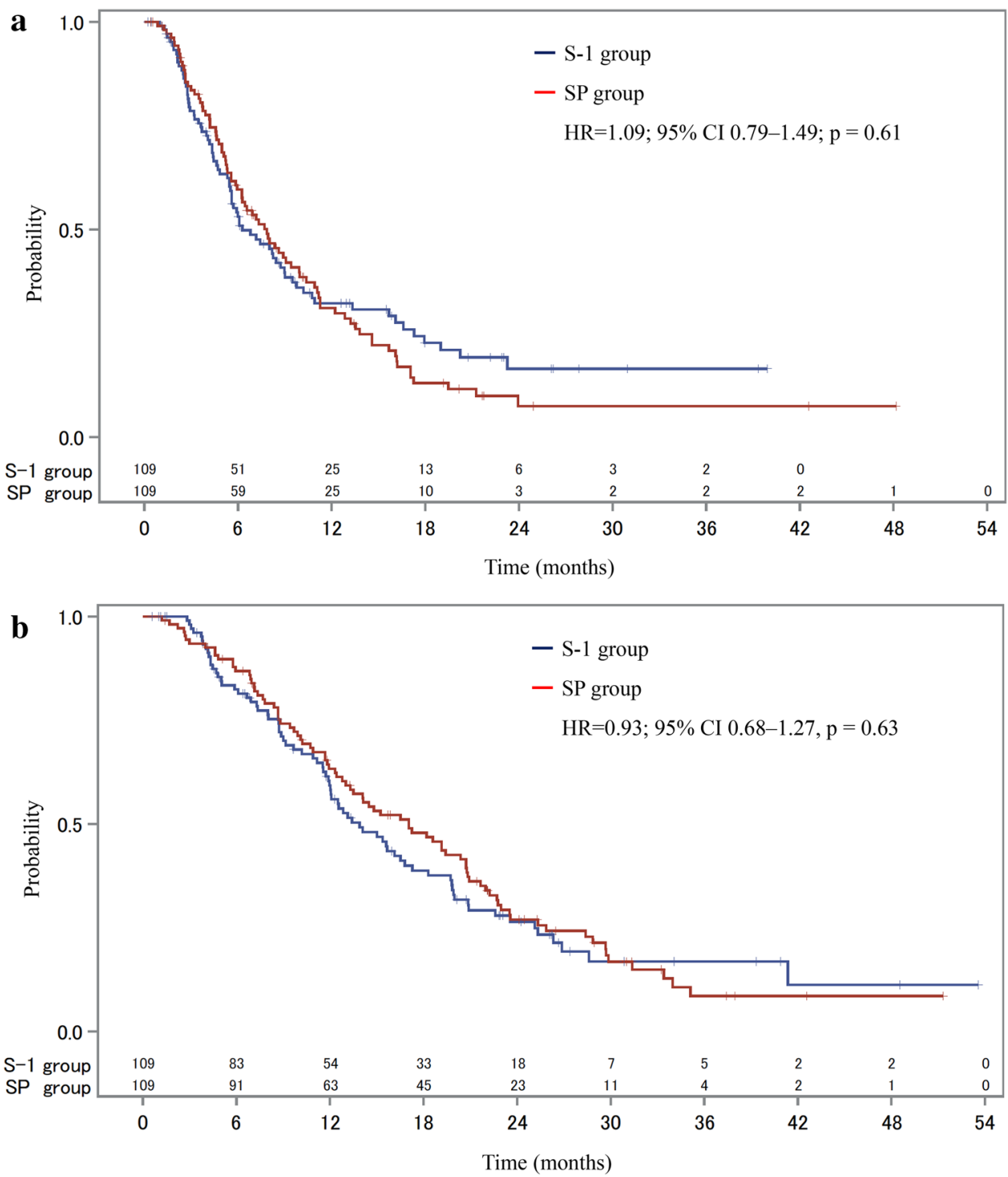

$86.9 \%$, and at 12 months was 59.3 and $63.4 \%$ in the S-1 and SP groups, respectively.

\section{Safety in the PSMC}

Any grade 3 or 4 AE was observed in $27.5 \%$ of the S-1 group patients and $49.5 \%$ of the SP group patients $(p<0.001)$ (Table 2). In the S-1 and SP groups, grade 3 or 4 leukopenia was observed in 1.8 and $11.0 \%(p=0.01)$, neutropenia in 6.4 and $20.2 \%(p<0.01)$, and anemia in 8.3 and $19.3 \%(p=0.02)$ of patients, respectively. In terms of non-hematological toxicity, grade 3 or 4 anorexia was more frequently observed in the SP group (19.3\%) than in the $\mathrm{S}-1$ group $(10.1 \%)$. Even though there were no treatment-related deaths in either treatment arms, hospitalization due to severe AEs occurred in $6.4 \%$ of patients in the S-1 group vs. $29.4 \%$ of patients in the SP group $(p<0.001)$. These observations strongly suggested that patients in the SP group experienced more severe AEs than those in the S-1 group.

\section{Multivariate analyses in the PSMC}

In the multivariate Cox proportional hazards regression analysis (Table 3), patients with a PS score 0 (vs. 1-2) (HR $0.65,95 \%$ CI $0.46-0.91, p=0.01)$ and normal range of C-reactive protein (CRP) (vs. abnormal range) (HR 0.61, 95\% CI $0.43-0.86, p<0.01)$ were associated with better OS. Treatment regimen was not significantly associated with better OS. Patients with a PS 0 (vs. 1-2) (HR 0.68, 95\% CI $0.50-0.93, p=0.01$ ), number of metastases $0-1$ (vs. 2 or more) (HR 0.67, 95\% CI 0.49-0.91, $p=0.01$ ) showed a significant correlation with favorable PFS. 
Table 2 Adverse events of the propensity score matched cohort

\begin{tabular}{|c|c|c|c|c|c|}
\hline & \multicolumn{2}{|l|}{ S-1 } & \multicolumn{3}{|l|}{ SP } \\
\hline & \multicolumn{2}{|c|}{$(n=109)$} & \multicolumn{3}{|c|}{$(n=109)$} \\
\hline & $\mathrm{G} \geq 3$ & $(\%)$ & $\mathrm{G} \geq 3$ & $\%$ & $p$ value \\
\hline Any $\mathrm{G} \geq 3$ & 30 & $(27.5)$ & 54 & $(49.5)$ & $<0.001$ \\
\hline \multicolumn{6}{|l|}{ Hematological } \\
\hline Leucopenia & 2 & $(1.8)$ & 12 & $(11.0)$ & 0.01 \\
\hline Neutropenia & 7 & $(6.4)$ & 22 & (20.2) & $<0.01$ \\
\hline Anemia & 9 & (8.3) & 21 & (19.3) & 0.02 \\
\hline Thrombocytopenia & 2 & $(1.8)$ & 6 & (5.5) & 0.28 \\
\hline \multicolumn{6}{|l|}{ Non-hematological } \\
\hline Nausea & 4 & (3.7) & 8 & (7.3) & 0.37 \\
\hline Anorexia & 11 & $(10.1)$ & 21 & (19.3) & 0.06 \\
\hline Diarrhea & 2 & $(1.8)$ & 6 & (5.5) & 0.28 \\
\hline Mucositis & 0 & $(0.0)$ & 2 & $(1.8)$ & 0.48 \\
\hline Fatigue & 3 & $(2.8)$ & 8 & (7.3) & 0.22 \\
\hline Febrile neutropenia & 1 & $(0.9)$ & 4 & (3.7) & 0.37 \\
\hline Creatinine increased & 0 & $(0.0)$ & 1 & $(0.9)$ & 1.00 \\
\hline Hospitalization due to $\mathrm{AE}$ & 7 & (6.4) & 32 & $(29.4)$ & $<0.001$ \\
\hline
\end{tabular}

$S P$ S-1 + cisplatin, $G$ CTCAE grade, $A E$ adverse event

Table 3 Multivariate analysis of predictive factors for OS and PFS in the propensity score matched cohort

\begin{tabular}{llllr}
\hline & HR & $95 \%$ CI & $p$ value \\
\hline Multivariate analysis for OS & & & & \\
$\quad$ PS 0 vs $\geq 1$ & 0.65 & 0.46 & 0.91 & 0.01 \\
CRP $\leq$ ULN vs $>$ ULN & 0.61 & 0.43 & 0.86 & $<0.01$ \\
Multivariate analysis for PFS & & & & \\
PS 0 vs $\geq 1$ & 0.68 & 0.50 & 0.93 & 0.01 \\
No. of metastasis $\leq 1$ vs $\geq 2$ & 0.67 & 0.49 & 0.91 & 0.01 \\
\hline
\end{tabular}

$O S$ overall survival, $P F S$ progression-free survival, $P S$ Eastern Cooperative Oncology Group performance status, $H R$ hazard ratio, $C I$ confidence interval

\section{Discussion}

In the present study, we collected clinical data from a large cohort of elderly AGC patients including both tolerable and intolerable for doublet chemotherapy. It was clear that the patients treated with S-1 alone were more fragile than those treated with SP in the originally collected patient cohort. Under such conditions, it could be easily expected that the patients of the SP group would show significantly longer OS than the patients of the S-1 group $(p=0.01)$ in the original entire cohort. Adopting the propensity score matching method to maximize the comparability, we compared the efficacy and safety between the S-1 monotherapy and SP groups in the patients who were considered to be tolerable to either regimen. As a result, there were no differences in TTF, PFS, and OS between the groups. It has been reported that a doublet chemotherapy of docetaxel plus cisplatin has failed to demonstrate survival advantages over docetaxel monotherapy in a phase III study (JCOG0803/WJOG4307L) for stage III, IV or recurrent non-small cell lung cancer patients aged 70 years or older [10]. Similarly, our results suggested that additional cisplatin might not be beneficial for survival of elderly patients with AGC.

In this study, SP showed a trend of higher response and disease control rates than $\mathrm{S}-1$ alone, whereas there were no significant differences in TTF, PFS, and OS between the two groups. This indicates that cisplatin works favorably for transient tumor shrinkage and stability, but may not contribute to prolongation of survival in elderly AGC. The duration of administration of cisplatin in the present study (3.7 months) was relatively shorter than that in the SPIRITS trial, in which the median number of cycles of SP was four cycles (corresponding to about 4.6 months) [6]. In addition, the initial dose of cisplatin was reduced in 30 patients $(27.5 \%)$ of the SP group. Although the precise relative dose intensity of each drug could not be obtained due to lack of precise information about drug delivery in both treatment groups of this study, short duration and/or decreased dose of cisplatin might result in no survival benefit over S-1 in our elderly patients with relatively good medical condition. The reasons for the treatment discontinuation were similar between the two groups, yet patients in the SP group were more likely to receive subsequent chemotherapy than those in the S-1 group. This may be associated with the higher response rate of the SP. It has been reported that the prognostic factors before the initiation of chemotherapy, such as PS, number of metastatic sites, and presence of peritoneal metastasis, affect survival after failure of the first-line chemotherapy [11]. These factors before the first-line chemotherapy were adjusted in the PSMC, but some unknown differences might remain in patients' physical condition. It was also unclear how the patients' socio-psychological condition after experiencing efficacy and toxicities in the first-line treatment influenced on the acceptance and willingness for subsequent therapy between the groups.

In the multivariate analysis of the PSMC, good PS, and normal serum ranges of CRP were shown to have strong correlations with the OS. Considering that PS has been generally recognized as the most important prognostic factor for OS in previous reports for patients with AGC [12, 13], CRP seems to be a specific prognostic factor in elderly patients with AGC because CRP is used in many other geriatric scores $[14,15]$. These factors were well balanced in our PSMC.

The incidence of severe AEs generally increased in the elderly patients because of lower organ function. It has been 
reported that severe AEs occur more within the SP group than within the S-1 group in previous retrospective studies [7, 8]. Furthermore, in this PSMC study, severe bone marrow suppression was more frequently observed in the SP group than in the S-1 group, and hospitalization due to AEs was astonishingly observed 5 times more. Although initial dose reduction rate of S-1 in the S-1 group was higher than in the SP group, it might be suggested that S-1 treatment is safer and more tolerable than the SP treatment in this PSMC. Based on these results, there could be no impact of adding cisplatin over S-1 from the aspect of both efficacy and safety for elderly patients with AGC.

There were two major limitations of the present study. The study cohort included a variety of patients with different clinical characteristics because of its retrospective nature, and could not exclude a selection bias of treating physicians in clinical decision, whether or which systemic chemotherapy could be administered to the elderly patients with AGC. The reasons for not using cisplatin with S-1 depended on the patients' background, such as age, PS, comorbidity, and renal function. Another limitation is the lack of information related to validate geriatric scores. Whether fit or unfit for intensive chemotherapy, patients cannot be evaluated only on the basis of chronological age. The geriatric assessment is needed because of the extreme diversity of the elderly population. The tolerability of chemotherapy for elderly patients is associated with renal function, medication, comorbidity, and family support [16-18]. These factors, in particular, hamper the development of a standard treatment for elderly patients.

Various therapeutic strategies for elderly patients with advanced malignant tumors have been attempted. However, they have not yet been established in many cancers, including AGC. Although doublet chemotherapy is regarded as standard of care worldwide, based on the results of the present study, we are now conducting a prospective comparative study for elderly patients with AGC (the WJOG8315G study) to clarify the role of geriatric assessment in optimizing treatment and identify a population more benefited from the study treatment.

In conclusion, our study using the propensity score matching method suggested that SP might have no survival benefits and cause more toxicities when compared with S-1 monotherapy for elderly patients with AGC. However, further investigation in this field is warranted.

Acknowledgements We thank all patients included in this study, the medical staff who contributed to the treatment of patients from each institution, and all the investigators in the West Japan Oncology Group (WJOG).

\section{Compliance with ethical standards}

Conflicts of interest No potential conflicts of interest to disclose.
Research involving human participants All procedures followed were in accordance with the ethical standards of the responsible committee on human experimentation (institutional and national) and with the Helsinki Declaration of 1964 and later versions. Informed consent or substitute for it was obtained from all patients for being included in the study.

\section{References}

1. Ferlay J, Bray F, Parkin DM, Pisani P, editors. Globocan 2000: cancer incidence and mortality worldwide (IARC Cancer Bases No. 5). Lyon: IARC Press; 2001.

2. Cancer Statistics in Japan. Foundation for promotion of cancer research. 2010. http://ganjoho.ncc.go.jp/public/statistics/back number/2010_en.html. Accessed 1 Oct 2015.

3. National Comprehensive Cancer Network. NCCN Clinical Practice Guidelines in Oncology. Older Adult Oncology (version 2. 2017).

4. National Comprehensive Cancer Network. NCCN Clinical Practice Guidelines in Oncology. Gastric Cancer (version 5.2017).

5. Boku N, Yamamoto S, Fukuda H, Shirao K, Doi T, Sawaki A, et al. Fluorouracil versus combination of irinotecan plus cisplatin versus $S-1$ in metastatic gastric cancer: a randomised phase 3 study. Lancet Oncol. 2009;10:1063-9.

6. Koizumi W, Narahara H, Hara T, Takagane A, Akiya T, Takagi M, et al. S-1 plus cisplatin versus S-1 alone for first-line treatment of advanced gastric cancer (SPIRITS trial): a phase III trial. Lancet Oncol. 2008;9:215-21.

7. Terazawa T, Iwasa S, Takashima A, Nishitani H, Honma Y, Kato $\mathrm{K}$, et al. Impact of adding cisplatin to $\mathrm{S}-1$ in elderly patients with advanced gastric cancer. J Cancer Res Clin Oncol. 2013;139:2111-6.

8. Tsushima T, Hironaka S, Boku N, Machida N, Yamazaki K, Yasui $\mathrm{H}$, et al. Comparison of safety and efficacy of S-1 monotherapy and S-1 plus cisplatin therapy in elderly patients with advanced gastric cancer. Int J Clin Oncol. 2013;18:10-6.

9. Eisenhauer EA, Therasse P, Bogaerts J, Schwartz LH, Sargent D, Ford R, et al. New response evaluation criteria in solid tumours: revised RECIST guideline (version 1.1). Eur J Cancer. 2009;45:228-47.

10. Abe T, Takeda K, Ohe Y, Kudoh S, Ichinose Y, Okamoto H, et al. Randomized phase III trial comparing weekly docetaxel plus cisplatin versus docetaxel monotherapy every 3 weeks in elderly patients with advanced non-small-cell lung cancer: the intergroup trial JCOG0803/WJOG4307L. J Clin Oncol. 2015;33:575-81.

11. Takashima A, Boku N, Kato K, Nakamura K, Mizusawa J, Fukuda $\mathrm{H}$, et al. Survival prolongation after treatment failure of first-line chemotherapy in patients with advanced gastric cancer: combined analysis of the Japan Clinical Oncology group trials JCOG9205 and JCOG9912. Gastric Cancer. 2014;17:522-8.

12. Takahari D, Boku N, Mizusawa J, Takashima A, Yamada Y, Yoshino T, et al. Determination of prognostic factors in Japanese patients with advanced gastric cancer using the data from a randomized controlled trial, Japan clinical oncology group 9912. Oncologist. 2014;19:358-66.

13. Takahari D, Mizusawa J, Koizumi W, Hyodo I, Boku N. Validation of the JCOG prognostic index in advanced gastric cancer of the SPIRITS and G-SOX trials, using individual patient data. Gastric Cancer. 2017;20:757-63.

14. Hubbard RE, O'Mahony MS, Calver BL, Woodhouse KW. Nutrition, inflammation, and leptin levels in aging and frailty. J Am Geriatr Soc. 2008;56:279-84. 
15. Walston J, McBurnie MA, Newman A, Tracy RP, Kop WJ, Hirsch $\mathrm{CH}$, et al. Frailty and activation of the inflammation and coagulation systems with and without clinical comorbidities: results from the Cardiovascular Health Study. Arch Intern Med. 2002;162:2333-41.

16. Balducci L, Extermann M. Cancer chemotherapy in the older patient: what the medical oncologist needs to know. Cancer. 1997;80:1317-22.
17. Boku N, Okamoto R, Kandabashi K, Sasaki E, Omuro Y, Maeda $\mathrm{Y}$, et al. Clinicopathological characteristics of gastric cancer in elderly patients. Gan To Kagaku Ryoho. 2006;33:1099-103.

18. Senden C, Vandecasteele T, Vandenberghe E, Versluys K, Piers $\mathrm{R}$, Grypdonck $\mathrm{M}$, et al. The interaction between lived experiences of older patients and their family caregivers confronted with a cancer diagnosis and treatment: a qualitative study. Int J Nurs Stud. 2015;52:197-206.

\section{Affiliations}

\section{Akitaka Makiyama ${ }^{1} \cdot K_{\text {Kenji Kunieda }}{ }^{2} \cdot$ Masaaki Noguchi $^{3} \cdot$ Takeshi Kajiwara $^{4} \cdot$ Takao Tamura $^{5} \cdot$ Koji Takeda $^{6}$. Junko Sugiyama ${ }^{7} \cdot$ Keiko Minashi $^{8} \cdot$ Toshikazu Moriwaki $^{9} \cdot$ Naotoshi Sugimoto $^{10} \cdot$ Michitaka Nagase $^{11}$. Yuji Negoro' ${ }^{12}$. Takashi Tsuda ${ }^{13}$. Hideki Shimodaira ${ }^{14} \cdot$ Naohiro Okano $^{15}$. Akihito Tsuji $^{16}$. Daisuke Sakai ${ }^{17}$. Kazuhiro Yanagihara $^{18}$. Shinya Ueda ${ }^{19}$. Shingo Tamura ${ }^{20}$. Satoshi Otsu' ${ }^{21} \cdot$ Takuya Honda $^{22}$. Yuzo Matsushita ${ }^{23}$. Tatsuya Okuno ${ }^{24}$. Tomomi Kashiwada ${ }^{25}$. Akira Nozaki ${ }^{26}$. Masahide Ebi' ${ }^{27} \cdot$ Hiroyuki Okuda $^{28}$. Mototsugu Shimokawa ${ }^{29}$. Shuichi Hironaka ${ }^{8}$. Ichinosuke Hyodo ${ }^{9} \cdot$ Eishi Baba $^{30} \cdot$ Narikazu Boku $^{31} \cdot$ Kei Muro $^{32}$. Taito Esaki ${ }^{33}$}

1 Department of Hematology/Oncology, Japan Community Healthcare Organization, Kyushu Hospital, 1-8-1 Kishinoura, Yahatanishi-ku, Kitakyushu 806-8501, Japan

2 Department of GI Oncology, Shizuoka Cancer Center, Shizuoka, Japan

3 Department of Gastrointestinal Oncology, National Cancer Center Hospital East, Kashiwa, Japan

4 Department of Gastrointestinal Medical Oncology, National Hospital Organization Shikoku Cancer Center, Matsuyama, Japan

5 Department of Medical Oncology, Kindai University Faculty of Medicine, Osaka-Sayama, Japan

6 Department of Medical Oncology, Osaka City General Hospital, Osaka, Japan

7 Department of Medical Oncology, Tonan Hospital, Sapporo, Japan

8 Clinical Trial Promotion Department, Chiba Cancer Center, Chiba, Japan

9 Division of Gastroenterology, Faculty of Medicine, University of Tsukuba, Tsukuba, Japan

10 Department of Medical Oncology, Osaka International Cancer Institute, Osaka, Japan

11 Department of Clinical Oncology, Nagoya Red-Cross Daiichi Hospital, Nagoya, Japan

12 Division of Gastroenterological Medicine, Kochi Health Sciences Center, Kochi, Japan

13 Department of Clinical Oncology, St. Marianna University School of Medicine, Kawasaki, Japan

14 Department of Clinical Oncology, Tohoku University Hospital, Sendai, Japan

15 Department of Medical Oncology, Kyorin University Faculty of Medicine, Tokyo, Japan

16 Department of Medical Oncology, Kagawa University Hospital, Kagawa, Japan
17 Department of Frontier Science for Cancer and Chemotherapy, Osaka University Graduate School of Medicine, Osaka, Japan

18 Department of Medical Oncology, Kansai Electric Power Hospital, Osaka, Japan

19 Department of Medical Oncology, Nara Hospital Kindai University School of Medicine, Ikoma, Japan

20 Department of Internal Medicine, Kyushu University Beppu Hospital, Beppu, Japan

21 Department of Medical Oncology and Hematology, Oita University Faculty of Medicine, Yufu, Japan

22 Department of Clinical Oncology Center, Nagasaki University Hospital, Nagasaki, Japan

23 Department of Medical Oncology, Hamanomachi Hospital, Fukuoka, Japan

24 Division of Gastroenterology, Department of Internal Medicine Graduate School of Medicine, Kobe University, Kobe, Japan

25 Division of Hematology, Respiratory Medicine and Oncology, Department of Internal Medicine, Faculty of Medicine, Saga University, Saga, Japan

26 Department of Clinical Oncology, National Hospital Organization Kyoto Medical Center, Kyoto, Japan

27 Department of Gastroenterology, Aichi Medical University School of Medicine, Nagakute, Japan

28 Department of Medical Oncology, Keiyukai Sapporo Hospital, Sapporo, Japan

29 Cancer Biostatistics Laboratory, Clinical Research Institute, National Hospital Organization Kyushu Cancer Center, Fukuoka, Japan

30 Department of Comprehensive Clinical Oncology, Faculty of Medical Sciences, Kyushu University, Fukuoka, Japan

31 Division of Gastrointestinal Medical Oncology, National Cancer Center Hospital, Tokyo, Japan 
32 Department of Clinical Oncology and Outpatient Treatment Center, Aichi Cancer Center Hospital, Nagoya, Japan
33 Department of Gastrointestinal and Medical Oncology, National Kyushu Cancer Center, Fukuoka, Japan 